\title{
Clinical evaluation of desensitizing treatments for cervical dentin hypersensitivity
}

\section{Ana Cecilia Corrêa Aranha ${ }^{(a)}$ Luiz André Freire Pimenta(b) Giselle Maria Marchi ${ }^{(c)}$}

(a) Assistant Professor, PhD, Department of Restorative Dentistry, School of Dentistry, University of São Paulo (USP), São Paulo, SP, Brazil.

(b) Clinical Professor, PhD, Department of Dental Ecology, School of Dentistry, University of North Carolina at Chapel Hill, Chapel Hill, North Carolina, USA

(c) Assistant Professor, PhD, Department of Restorative Dentistry, Piracicaba Dental School, University of Campinas (UNICAMP), Piracicaba, SP, Brazil.

\section{Corresponding author:}

Ana Cecilia Corrêa Aranha

Depto. de Dentística Restauradora,

Faculdade de Odontologia da USP

Av. Prof. Lineu Prestes, 2227, Cid.

Universitária

São Paulo - SP - Brazil

CEP: 05508-900

E-mail: acca@usp.br

Received for publication on Apr 17, 2008

Accepted for publication on Aug 22, 2008

\begin{abstract}
The aim of this study was to compare different treatments for dentin hypersensitivity in a 6-month follow-up. One hundred and one teeth exhibiting non carious cervical lesions were selected. The assessment method used to quantify sensitivity was the cold air syringe, recorded by the visual analogue scale (VAS), prior to treatment (baseline), immediately after topical treatment, after 1 week, 1, 3 and 6 months. Teeth were randomly assigned to five groups $(n=20)$ : G1: Gluma Desensitizer (GD); G2: Seal\&Protect (SP); G3: Oxa-gel (OG); G4: Fluoride (F); G5: Low intensity laser-LILT $\left(660 \mathrm{~nm} / 3.8 \mathrm{~J} / \mathrm{cm}^{2} / 15 \mathrm{~mW}\right)$. Analysis was based on the non-parametric Kruskal-Wallis test that demonstrated statistical differences immediately after the treatment $(\mathrm{p}=0.0165)$. To observe the individual effects of each treatment, data was submitted to Friedman test. It was observed that GD and SP showed immediate effect after application. Reduction in the pain level throughout the six-month follow-up was also observed. In contrast, LILT presented a gradual reduction of hypersensitivity. OG and F showed effects as of the first and third month respectively. It can be concluded that, after the 6-month clinical evaluation, all therapies showed lower VAS sensitivity values compared with baseline, independently of their different modes of action.
\end{abstract}

Descriptors: Dentin hypersensitivity; Gels; Laser therapy, low-level; Patient outcome assessment. 


\section{Introduction}

Dentin hypersensitivity is a common complaint and it is one of the most painful and least successfully resolved problems of the teeth., ${ }^{1,2}$ It is defined as a short and sharp pain arising from exposed dentin, in response to chemical, thermal, tactile or osmotic stimuli, that cannot be explained as arising from other forms of old dental defect or pathology..$^{1-3}$

There are many and varied etiological and predisposing factors related to dentin hypersensitivity. Removal of enamel, as a result of attrition, abrasion and erosion, or denudation of the root surface by loss of the overlying cementum and periodontal tissues is commonly cited. ${ }^{2}$

As exposure of the root area may be multifactorial, chronic trauma from tooth brushing, tooth flexure due to abnormal occlusal loading forces, parafunctional habits, acute and chronic inflammatory gingival and periodontal diseases, acute trauma, periodontal surgery, and acidic dietary components, are commonly cited as major causes of cervical lesions and dentin hypersensitivity. ${ }^{4}$

Microscopically, the features that determine the degree of hypersensitivity in subjects include the number, diameter and size of the open dentinal tubules. In sensitive teeth, the number of tubules per unit area is about eight times greater than the number found in non-sensitive teeth, and the tubular diameter is two times greater. ${ }^{5}$

Numerous desensitizing agents have been clinically tested over several decades in an effort to alleviate discomfort from cervical dentin hypersensitivity. ${ }^{3,4,6-8}$ Results have been variable and to some extent inconclusive, due to the different methodologies employed, variability of the subjective response and the influence of a placebo effect.

Thus, the purpose of this study was to investigate the clinical efficacy of some desensitizer agents in reducing cervical dentin hypersensitivity over a 6 month period.

\section{Materials and Methods}

The research protocol was initially submitted to the Ethics Committee of the School of Dentistry at Piracicaba, Brazil. Patients who gave their oral and voluntary written informed consent and were aware of the study inclusion and exclusion criteria were examined prior to entry into the study. A detailed medical and dental history was recorded by examiner 1 to rule out certain participants. Patients were considered suitable for the study if they had sensitive teeth showing abrasion, erosion or recession with exposure of cervical dentin. Teeth with evidence of pulpitis, carious lesions, defective restorations, facets of attrition, premature contact, cracked enamel, active periodontal disease, daily doses of medications or any factor that could be responsible for sensitivity complaints, were also excluded. Other exclusion criteria were professional desensitizing therapy during the previous 3 months; neither pregnant nor lactating women were recruited.

After clinical examination, thirty-nine patients (101 teeth) were selected. Of these teeth, 68.3\% were premolars, $14.8 \%$ were canines, $9.9 \%$ were incisors and $6.9 \%$ were molars. All lesions were located in the facial surface of the teeth.

If the patient had two lesions side-by-side in the same quadrant, just one of the lesions would receive the treatment at that moment. So, all patients would have at least one lesion per quadrant to be treated. Comparisons were made between the treatments, as it was difficult to subject the patients to the five desensitizing methods provided.

In the first screening visit, non-fluoride toothpaste Phillips (SmithKline Beecham, Brentford, UK) and soft toothbrush (Colgate-Palmolive, São Paulo, SP, Brazil) were dispensed for home use during the period of the study. Dietary counseling and oral hygiene instructions techniques were also provided.

The week before treatment, all patients were standardized and the lesions were randomly assigned to the groups. Dentin hypersensitivity was assessed by examiner 1 through a cold air stimulus. The subject's response was considered as a baseline measurement (PRE-1), according to the visual analogue scale of pain (VAS). Each patient was asked to rate the perception of discomfort after the application of air by a dental syringe at 45 to $60 \mathrm{psi}, 2 \mathrm{~mm}$ away from and perpendicular to the root surface for 3 seconds. Neighboring teeth were isolated during testing using the operator's fingers and cotton rolls. The VAS scale consists of a horizontal line, $100 \mathrm{~mm}$ 
long, anchored at the left end by the descriptor "no pain" and at the other end by "unbearable pain." The patients were asked to rate their pain according to the scale in order to mark the severity of their hypersensitivity. The distance of this point in millimeters from the left end of the scale was recorded and used as the VAS score. Patients were accepted entry into the study with a VAS score $\geq 40 \mathrm{~mm} .^{9}$

Five minutes after the first measurement, patients received the treatment according to the manufacturers' instructions (Table 1), by examiner 2 . No negative control group was allowed by the Ethics Committee.

Prior to the topical application of the desensitizing agents, the area received oral prophylaxis with pumice and was isolated with cotton rolls.

\section{Group 1 - Gluma Desensitizer}

A few drops of Gluma Desensitizer (Heraeus Kulzer, Armonk, NY, USA) were applied with a cotton pellet using a gentle but firm rubbing motion. After 30 seconds, the area was dried thoroughly until the fluid disappeared and the surface was not shinny.

\section{Group 2 - Seal\&Protect}

A few drops of Seal\&Protect (Dentsply, Petrópolis, RJ, Brazil) were applied to the dentin surface with an applicator tip. The surface was left undisturbed for 20 seconds and the excess solvent removed by gently airing for a few seconds and cured for 10 seconds. With a cotton pellet, the oxygen-inhibited layer was removed and the excess checked with a periodontal probe.

\section{Group 3 - Oxa-Gel}

The $3 \%$ potassium oxalate gel (Oxa Gel, Art Dent, Araraquara, SP, Brazil) was applied to the dentin surface with a cotton pellet and left undisturbed for 2 minutes. After that, only the excess was removed.

\section{Group 4 - Acidulated Phosphate Fluoride}

The gel form of Acidulated Phosphate Fluoride (Nuprogel, Dentsply, Petrópolis, RJ, Brazil) was applied for 1 minute. Excess gel was removed with a cotton pellet and the patients were advised not to drink or eat for the next hour after the application of the product.

\section{Group 5 - Low Intensity Laser Therapy (LILT)}

The equipment used was a low power laser of GaAlAs semiconductor laser diode (MMOptics, São Carlos, SP, Brazil) operating at a continuous wavelength of $660 \mathrm{~nm}$ and power of $15 \mathrm{~mW}$ at an energy level of $3.8 \mathrm{~J} / \mathrm{cm}^{2}$, following the protocol of the Special Laboratory of Lasers in Dentistry (LELO) at the University of São Paulo.

The irradiation method involved three different irradiation points in the exposed dentin (mesial, distal and central surfaces of the lesion) and one point in the tooth apex, each one lasting 10 seconds, in contact mode. This procedure was repeated three times, with intervals of 3 days between them.

Five minutes after finishing the procedure, the level of hypersensitivity was quantified and the data was described as POST-1. For group 5, after the

Table 1 - Manufacturers and composition of the desensitizing products.

\begin{tabular}{c|l|l|l}
\hline Group & \multicolumn{1}{|c|}{ Material } & \multicolumn{1}{|c}{ Manufacturer } & \multicolumn{1}{c}{ Active ingredients } \\
\hline 1 & Gluma Desensitizer & Heraeus Kulzer Inc., Armonk, NY, USA & $5 \%$ glutaraldehyde and 35\% hydroxyethyl methacrylate (HEMA) \\
\hline 2 & Seal\&Protect & Dentsply, Petrópolis, RJ, Brazil & $\begin{array}{l}\text { Di- and trimethacrylate resins, PENTA, silica, triclosan, } \\
\text { cetylamine hydrofluoride and acetone }\end{array}$ \\
\hline 3 & Oxa-gel & Art Dent, Araraquara, SP, Brazil & $3 \%$ potassium oxalate, pH 4 \\
\hline 4 & $\begin{array}{l}\text { Acidulated Phosphate } \\
\text { Fluoride (Nuprogel) }\end{array}$ & Dentsply, Petrópolis, RJ, Brazil & $2.59 \%$ sodium fluoride, 1.16\% phosphoric acid \\
\hline 5 & Low Intensity Laser & MMOptics, São Carlos, SP, Brazil & Semiconductor diode laser of GaAIAs (660 nm) \\
\hline
\end{tabular}


third irradiation section, the sensitivity was quantified as POST-1.

The effectiveness of the therapies was assessed by examiner 1 at the five examination periods: immediately after the application of the desensitizing agent (POST-1), after 1 week (POST-2), 1 month (POST3), 3 months (POST-4) and 6 months (POST-5).

\section{Results}

For comparing the effectiveness of the treatments, teeth were used as a statistical unit rather than a subject. Data was submitted to the non-parametric Kruskal-Wallis analysis of variance and multiple comparison tests with the level of significance of $5 \%$. Comparing the desensitizing treatments, the statistical analysis revealed significant differences between the periods of examination. Statistical differences were observed immediately after the treatment $(\mathrm{p}=0.0165)$. Oxa-Gel and LILT showed the higher scores of sensitivity when compared to Gluma Desensitizer and Seal\&Protect. Acidulated Phosphate Fluoride presented an intermediary level of sensitivity.

To observe the individual effect of each treatment, data was submitted to Friedman Analysis of Variance Test $(\mathrm{p}=0.000)$. Reduction of sensitivity was significant for all treatments. Table 2 and Graph 1 indicate the mean scores for treatment at different time intervals.

It was observed that Gluma Desensitizer and Seal\&Protect showed immediate effect after application and no statistically significant differences were observed between the two therapies. The sensitivity level was kept the same until the end of the study. Regarding irradiation with LILT, the ef- fectiveness was not immediate. The sensitivity level dropped in the first week of evaluation, remaining constant until the end. The desensitizer agents OxaGel and APF gel showed effects as of the first and third months respectively.

\section{Discussion}

In the present study, it was noted that independently of the treatment, after six months of clinical follow up, all desensitizing agents were capable of reducing dentin hypersensitivity, presenting no statistically significant differences on the Post- 5 scale.

Considerable evidence has been accumulated to support the hydrodynamic theory. ${ }^{10,11}$ This theory proposes that stimulus on the exposed dentin surface causes a displacement of the fluid inside the tubules that activates the nerve terminals in the dentin

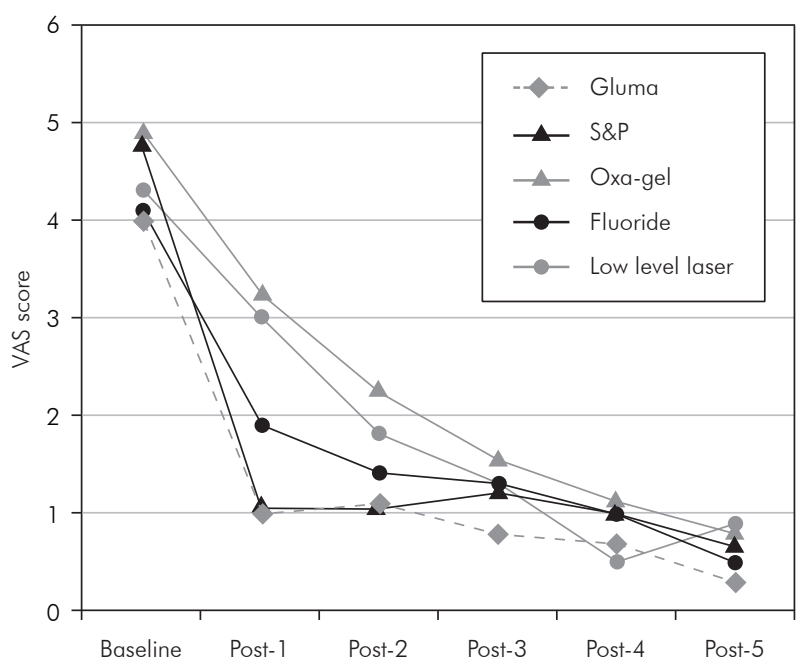

Graph 1 - Illustrative representation of mean scores for treatment at different time intervals.

Table 2 - Baseline and posttreatment mean visual analogue scale values.

\begin{tabular}{l|c|c|c|c|c}
\hline & Gluma & S\&P & Oxa-Gel & APF & Laser \\
\hline Baseline & $4.0 \mathrm{~A}$ & $4.75 \mathrm{~A}$ & $4.90 \mathrm{~A}$ & $4.1 \mathrm{~A}$ & $4.3 \mathrm{~A}$ \\
\hline Post-1 (5 min) & $1.0 \mathrm{~B}$ & $1.05 \mathrm{~B}$ & $3.25 \mathrm{~A}$ & $1.9 \mathrm{~A}$ & $3.0 \mathrm{~A}$ \\
\hline Post-2 (1 week) & $1.1 \mathrm{~A}$ & $1.05 \mathrm{~A}$ & $2.25 \mathrm{~A}$ & $1.4 \mathrm{~A}$ & $1.8 \mathrm{~A}$ \\
\hline Post-3 (1 month) & $0.8 \mathrm{~A}$ & $1.20 \mathrm{~A}$ & $1.55 \mathrm{~A}$ & $1.3 \mathrm{~A}$ & $1.3 \mathrm{~A}$ \\
\hline Post-4 (3 months) & $0.7 \mathrm{~A}$ & $1.00 \mathrm{~A}$ & $1.15 \mathrm{~A}$ & $1.0 \mathrm{~A}$ & $0.5 \mathrm{~A}$ \\
\hline Post-5 (6 months) & $0.3 \mathrm{~A}$ & $0.65 \mathrm{~A}$ & $0.80 \mathrm{~A}$ & $0.5 \mathrm{~A}$ & $0.9 \mathrm{~A}$ \\
\hline
\end{tabular}

*Similar letters in a horizontal line imply no statistical significant differences. APF: Acidulated phosphate fluoride. 
and pulp, causing pain. Taking into consideration that the application of desensitizing agents is a noninvasive treatment and also its potential in reducing the fluid movement through the narrowing or occlusion of tubule openings, its use is strongly recommended as observed in the literature. ${ }^{6-8}$

However, the advent of dental lasers has raised another option for the treatment of dentinal hypersensitivity and has become a research interest in the last decades. ${ }^{12-15}$ In the present study, laser therapy provided a considerable decrease in sensitivity level; however, the response was slower when compared to the effect of desensitizing agents.

Although information on the neurophysiologic mechanism is not conclusive, it is postulated that a low intensity laser mediates an analgesic effect related to the depolarization of C-fiber afferents. This interference in the polarity of cell membranes by increasing the amplitude of the action potential of cell membranes can block the transmission of pain stimuli in hypersensitive dentin. ${ }^{14}$

Due to the lack of information related to the irradiation protocol used and the subjectivity of the evaluation of dentin hypersensitivity, contradictory results are reported in the literature. ${ }^{12}$

It is worth emphasizing, furthermore, that although the mechanisms of low intensity laser activity are still not clear, the results obtained in this study may have occurred due to the biomodulation effect of the irradiation. Histological studies have reported that hard tissue formation is enhanced as a reaction of dental pulp to laser light. ${ }^{16,17}$ In the present study, the non-immediate effect of Low Intensity Laser, but gradual reduction in sensitivity over a period of 6 months can explain the biomodulation effect.

Although speculative, the mechanisms proposed for the effects of low intensity laser require serious considerations and new experiments. It can be stated that the diode laser is an effective method for the treatment of dentin hypersensitivity, considering the treatment to be predictable, reliable and simple.

With regard to Gluma Desensitizer and Seal\&Protect, both desensitizers showed an immediate effect after application and the level of sensitivity remained the same until the 6-month period.

The Gluma Desensitizer product contains 5\% glutaraldehyde and 35\% hydroxyethyl methacrylate (HEMA). The hypothesis for the immediate occlusion of the dentin tubules is an effect of glutaraldehyde on the proteins of the dentinal fluid. In the reaction of glutaraldehyde with dentin, the two groups of aldehydes present in glutaraldehyde interlace themselves with the amino groups of dentin collagen, leading to a fixing of proteins, forming a barrier. ${ }^{18,19}$ The positive results of Gluma Desensitizer presented in this study are in agreement with the literature. ${ }^{6,7,20,21}$

The desensitizing agent Seal\&Protect showed similar results to those shown by Gluma. The agent Seal\&Protect is derived from the adhesive system Prime\&Bond NT that has an anti-microbial characteristic, resulting from the incorporation of triclosan, and acid monomers, which are self-conditioning. ${ }^{8,22}$

Considering the desensitizing effect of Oxa-Gel, it did not differ statistically in relation to the baseline up to the first month after application. As of the first month, a gradual reduction in sensitivity levels was noted until the six-month evaluation term. In the literature, the solution of $3 \%$ monohydrogenated-monopotassium $(\mathrm{pH} 2)$ acts as a weak dentin acid conditioner, increasing the concentration of ionized calcium to extremely high levels, resulting in an accelerated formation of crystals. However, in spite of the satisfactory results found in literature ${ }^{23-}$ ${ }^{26}$, it is reported that water spray can remove the oxalate crystals on the dentin surface, because the desensitizer agent is short-lived. ${ }^{27,28}$

The desensitizing effect of potassium is also related to the inactivation of nerve fibers. This double action of potassium oxalate may increase its possibility of combining therapies, both physical by tubular occlusion and neural by depolarizing the membrane. ${ }^{29}$

The non-immediate effect of potassium oxalate could be compared to the APF gel agent. Statistically significant differences in the level of sensitivity were detected as of the third month, suggesting an interference of the placebo effect.

In contact with the mineralized structures, the fluoridated substances react chemically with the calcium and phosphate ions providing a precipitation of $\mathrm{CaF}_{2}$ crystals. $^{28,30}$ Because it is an unstable compound, $\mathrm{CaF}_{2}$ rapidly dissociates after applica- 
tion, so that the anti-hyperesthesia effect is of short duration. In spite of fluoride being recognized as an effective anti-caries agent, its use as a desensitizing agent is still reported as unsuccessful when compared to therapeutic agents such as Gluma or Seal\& Protect, despite its distinct modes of action.

When evaluating the results of this study, it should be mentioned that the teeth were used as the unit of analysis. This might not be the most appropriate way to analyze the data considering the potential effects of study participation, especially in a pain-related study; however, it enables the research to assess as many different products with a smaller number of patients.

It should be considered that the evaluation of treatments for dentin hypersensitivity is not a simple procedure due to the interference of the placebo effect, the natural desensitization of the dentin, and the mechanical occlusion of the dentin tubules by smear layer or secondary dentin..$^{6,8,12,20,30}$ Because it is a painful and subjective phenomenon, the pain from the cervical lesion may be modified by the subject's emotional components. ${ }^{23,24}$

\section{References}

1. Addy M, Urquhart E. Dentine hypersensitivity: its prevalence, aetiology and clinical management. Dent Update. 1992;19(10):407-8, 410-2.

2. Walters PA. Dentinal hypersensitivity: a review. J Contemp Dent Pract. 2005;6(2):107-17.

3. Canadian Advisory Board on Dentin Hypersensitivity. Consensus-based recommendations for the diagnosis and management of dentin hypersensitivity. J Can Dent Assoc. 2003;69(4):221-8.

4. Chabanski MB, Gillam DG. Etiology, prevalence and clinical features of cervical dentin sensitivity. J Oral Rehabil. 1997;24(9):15-9.

5. Yoshiyama AM, Suge T, Kawasaki A, Ebisu S. Morphological characterization of tubule-like structures in hypersensitive human radicular dentin. J Dent. 1996;24(1-2):57-63.

6. Kakaboura A, Rahiotis C, Thomaidis S, Doukoudakis S. Clinical effectiveness of two agents on the treatment of tooth cervical hypersensitivity. Am J Dent. 2005;18(4):291-5.

7. Olusile AO, Bamise CT, Oginni AO, Dosumu OO. Short-term clinical evaluation of four desensitizing agents. J Contemp Dent Pract. 2008;9(1):22-9.
Taking into consideration the effectiveness of the agents used, it is observed that each agent has advantages and disadvantages, in relation to cost and time consumption. Opting for a desensitizing agent, the factors that lead to dentin exposure and hypersensitivity should be controlled by means of guidance on diet and brushing, and also occlusal adjustment, in order for an efficient treatment to be carried out.

\section{Conclusion}

After the 6-month follow-up period, it was possible to conclude that hypersensitive dentin is a challenging condition that involves specific approaches and a multidisciplinary treatment. All therapies studied in the present study showed lower VAS sensitivity values compared with baseline, independently of their different modes of action.

\section{Acknowledgments}

The authors would like to thank Prof. Gláucia Ambrosano for her support in the statistical analysis.

8. Pamir T, Dalgar H, Onal B. Clinical evaluation of three desensitizing agents in relieving dentin hypersensitivity. Oper Dent. 2007;32(6):544-8.

9. Clarck GE, Troullos ES. Designing hypersensitivity clinical studies. Dent Clin North Am. 1990;34(3):531-44.

10. Brännström M. Sensitivity of dentin. Oral Surg Oral Med Oral Pathol. 1966;21(4):517-27.

11. Gillam DG. Mechanisms of stimulus transmission across dentin - a review. J West Soc Periodontol Periodontal. 1995;43(2):53-65.

12. Benetti AR, Franco EB, Franco EJ, Pereira JC. Laser Therapy for dentin hypersensitivity: a critical appraisal. J Oral Laser Appl. 2004;4:271-8.

13. Gerschman JA, Ruben J, Gebart-Eaglamont J. Low level laser therapy for dentinal tooth hypersensitivity. Aust Dent J. 1994;39(6):353-7.

14. Kimura Y, Wilder-Smith P, Yonaga K, Matsumoto K. Treatment of dentin hypersensitivity by lasers: a review. J Clin Periodontol. 2000;27(10):715-21.

15. Marsilio AL, Rodrigues JR, Borges AB. Effect of the Clinical application of the GaAlAs laser in the treatment of dentin hypersensitivity. J Clin Laser Med Surg. 2003;21(5):291-6. 
16. Ferreira AN, Silveira L, Genovese WJ, de Araújo VC, Frigo $\mathrm{L}$, de Mesquita RA et al. Effect of GaAIAs laser on reactional dentinogenesis induction in human teeth. Photomed Laser Surg. 2006;24(3):358-65.

17. Matsui S, Tsujimoto Y, Matsushima K. Stimulatory effects of hydroxyl radical generation by Ga-Al-As laser irradiation on mineralization ability of human dental pulp cells. Biol Pharm Bull. 2007;30(1):27-31.

18. Dijkman GEHM, Jonebloed WL, de Vries J, Ogaard B, Arends J. Closing of dentinal tubules by Glutaraldeyde treatment, a scanning electron microscopy study. Scand Dent Res. 1994;102(3):144-50.

19. Schüpbach P, Lutz F, Finger WJ. Closing of dentinal tubules by Gluma Desensitizer. Eur J Oral Sci. 1997;105(5):414-21.

20. Camps J, Pizzanti S, Dejou J, Franquin JC. Effects of desensitizing agents on human dentin permeability. Am J Dent. 1998;1(6):286-90.

21. Davidson DF, Suzuki M. The Gluma bonding System: a clinical evaluation of its various components for the treatment of hypersensitive root dentin. J Can Dent Assoc. 1997;63(1):3841.

22. Azzopardi A, Bartlett DW, Watson TF, Sherriff M. The measurement and prevention of erosion and abrasion. J Dent. 2001;29(6):395-400.
23. Cooley RL, Sandoval VA. Effectiveness of potassium oxalate treatment on dentin hypersensitivity. Gen Dent. 1989;37(4):330-3.

24. Gillam DG, Mordan NJ, Sinodinou AD, Tang JY, Knowles JC. The effects of oxalate-containing products on the exposed dentin surface: an SEM investigation. J Oral Rehabil. 2001;28(11):1037-44.

25. Muzzin KB, Johnson R. Effects of potassium oxalate on dentin hypersensitivity in vivo. J Periodontol. 1989;60(3):151-8.

26. Pashley DH, Galloway SE. The effects of oxalate treatment on the smear layer of ground surfaces of human dentin. Arch Oral Biol. 1985;30(10):731.

27. Kerns DG, Scheidt MJ, Pashley DH, Horner JA, Strong SL, Van Dyke TE. Dentinal tubule occlusion and root hypersensitivity. J Periodontol. 1991;62(7):421-8.

28. Pereira JC, Segala AD, Gillam DG. Effect of desensitizing agents on the hydraulic conductance of human dentin subjected to different surface pre-treatments - an in vitro study. Dent Mater. 2005;21(2):129-38.

29. Orchardson R, Gillam DG. The efficacy of potassium salts as agents for treating dentin hypersensitivity. J Orofac Pain. 2000;14(1):9-19.

30. Yates RJ, Newcombe RG, Addy M. Dentine hypersensitivity: a randomized, double-blind placebo-controlled study of the efficacy of a fluoride-sensitive teeth mounthrise. J Clin Periodontol. 2004;31(10):885-9. 